Sociologie et sociétés

\title{
Les appareils militaires dans les formations sociales dépendantes d'Amérique latine
}

\section{The Military Apparatus in Latin American Dependent Social} Organization

\section{Los aparatos militares en las formaciones sociales dependientes de América latina}

\section{Robert VANDYCKE}

Volume 3, numéro 2, novembre 1971

URI : https://id.erudit.org/iderudit/001823ar

DOI : https://doi.org/10.7202/001823ar

Aller au sommaire du numéro

Éditeur(s)

Les Presses de l'Université de Montréal

ISSN

0038-030X (imprimé)

1492-1375 (numérique)

Découvrir la revue

Citer cet article

VANDYCKE, R. (1971). Les appareils militaires dans les formations sociales dépendantes d'Amérique latine. Sociologie et sociétés, 3(2), 175-188. https://doi.org/10.7202/001823ar
Résumé de l'article

Après avoir montré l'impossibilité d'analyser le rôle politique des militaires latino-américains à partir de leur origine sociale ou de leur appartenance à la bureaucratie urbaine, l'auteur propose de considérer l'armée comme appareil d'État. Cependant, dans une situation de dépendance, l'aide militaire étrangère vient perturber les mécanismes de contrôle de la classe dominante, sur l'appareil militaire. L'examen de ces deux aspects - appareil d'État et aide étrangère - permet de comprendre la marge d'autonomie de l'armée ainsi que le sens et les limites de son rôle politique, compte tenu des classes et de leurs rapports dans les formations latino-américaines. 


\section{Les appareils militaires dans les formations sociales dépendantes d'Amérique latine}

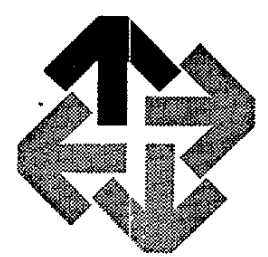

ROBERT VANDYCKE

Les études SUR Les interventions militaires en Amérique latine sont souvent: entachées de jugements de valeur, de formalisme ou encore, souffrent d'une grande faiblesse sur le plan de l'explication ${ }^{1}$. Deux recherches récentes présentent cependant des hypothèses très intéressantes dont l'examen critique offre des points de repère utiles : il s'agit des travaux de José Nun sur les coups d'État et d'Alain Joxe sur l'armée chilienne ${ }^{2}$. Les insuffisances relatives de ces théories conduisent cependant à proposer le recours aux concepts d'appareil d'État et de dépendance comme susceptibles d'éclairer quelque peu le phénomène, malgré sa complexité et la rareté des données objectives disponibles.

1. On peut trouver un compte rendu des principaux ouvrages parus sur la question dans L. N. McAlister, * Recent Research and Writings on the Role of the Military in Latin America, ", Latin American Research Review, vol. II, $\mathbf{n}^{\circ}$ 1, 1966, p. 5-36.

2. José Nun, "The Middle Class Military Coup \#, in Claudio Veliz (édit.), The Politics of Conformity in Latin America, New York, Oxford University Press, 1967, p. 66-118. (L'article légèrement abrégé est disponible en français dans Sociologie du travail, $\mathrm{n}^{\mathrm{p}} 3,1967$.) Alain Joxe, las Fuerzas armadas en el sistema politico de Chile, Santiago, Editorial Universitaria, 1.970. Pour les effectifs et équipements des armées latino-américaines, nous renvoyons à : "Les forces armées mondiales en 1970 », Notes et études documentaires. La documentation française, $\mathrm{n}^{\mathrm{os}} 3812,3813,3814,12$ septembre 1971, spécialement p. 81-92. 


\section{JOSÉ NUN OU L'ORIGINE SOCIALE DES MILITAIRES}

L'intérêt de Nun porte, contrairement à de nombreux auteurs, moins sur le fait que sur le sens de l'immixtion des militaires dans le domaine politique. Son interprétation souligne la représentation des aspirations de la classe moyenne ${ }^{3}$ par l'armée, du moins dans les pays les plus riches, les plus urbanisés de l'Amérique latine, et ceux où la classe moyenne est nombreuse, comme au Brésil, en Argentine, etc. ${ }^{4}$.

La thèse de l'auteur a le grand mérite de détruire la conception simpliste courante selon laquelle le développement des «secteurs moyens » serait un facteur important de stabilité du régime politique. En posant la condition dans laquelle on peut envisager cette relation classe moyenne-stabilité (la vocation hégémonique de cette classe montante), Nun en renverse le signe pour ce qui a trait à l'Amérique latine : l'armée intervient précisément en raison des valeurs qui la lient à ces secteurs moyens inorganisés, incapables de s'affirmer face au projet hégémonique dépassé de l'oligarchie et enfermés dans des revendications économico-corporatistes.

Cette thèse ne peut cependant être acceptée sans réserve. Tout d'abord, l'auteur note lui-même une exception : le renversement d'Irigoyen par un coup d'État en Argentine, malgré sa politique favorable à la promotion des classes moyennes. Ce cas particulier appellerait à lui seul des hypothèses qui nuanceraient le lien trop mécanique établi par l'auteur entre les militaires et la classe moyenne. Or, Nun s'attache plutôt à réfuter l'argumentation basée sur l'armée comme institution totalitaire ${ }^{5}$ et sur l'importance de la socialisation. Pour lui, cette dernière passe au second plan; l'activité politique incessante du militaire a ouvert l'armée aux influences externes et d'ailleurs, au dire même de Goffmann, la victoire culturelle n'est pas le but réel de l'institution totalitaire ${ }^{6}$. Bref, l'explication du comportement politique des officiers résiderait beaucoup moins dans leur formation que dans les valeurs et aspirations correspondant à leur origine sociale : la classe moyenne.

On n'entamera pas ici une discussion sur la portée des différences entre les armées latino-américaines et le type idéal de l'institution totalitaire, compte tenu de ce qui constitue l'unité significative de ce dernier. On concédera même à Nun que, dans un pays comme le Brésil, les relations entre industriels et militaires ont atteint un tel degré d'intégration qu'un auteur a pu évoquer la constitution d'un complexe militaire industriel ${ }^{7}$. Il ne faudrait cependant pas trop minimiser les effets d'un endoctrinement qui porte de plus en plus sur les facteurs sociopolitiques du maintien de l'ordre intérieur.

3. L'auteur englobe dans la classe moyenne la bourgeoisie qui est en expansion mais qui s'intègre dans le cadre de l'hégémonie oligarchique plutôt que de s'y opposer et de chercher à lui substituer le sien propre. Ce point de vue ne fait pas l'unanimité : voir notamment $A$. Joxe, las Fuerzas armadas en el sistema politico de Chile, et Braun, in Revista latinoame، icana de sociologia, $\mathrm{n}^{\circ} 1,1969, \mathrm{p} .134$.

4. Ces critères de classification des sociétés latino-américaines prêtent à discussion, mais ils sont cohérents avec sa thèse.

5. Voir Erving Goffmann, Asiles, Paris, Editions de Minuit, 1968.

6. Cette argumentation de Nun est développée dans "The Middle Class Military Coup », in Claudio Veliz (édit.), op. cit., p. 73-74.

7. Octavio Ianni, O colapso do populismo no Brasil, Rio, Civilizaçâo Brasileira, 1968, p. 204-207. 
Convaincante lorsqu'il s'agit de détruire la relation automatique entre les intérêts de l'oligarchie et les coups d'État, la démonstration de Nun est plus discutable en ce qui concerne les liens entre l'organisation militaire et les classes moyennes. Car si les rares études empiriques sur l'origine sociale des haut gradés ${ }^{8}$ montrent que ceux-ci proviennent depuis longtemps, en Argentine, de milieux divers et spécialement des classes moyennes urbaines, ces mêmes recherches font cependant ressortir une représentation très honorable de l'oligarchie. De plus, en admettant que la référence de l'armée aux valeurs de la classe moyenne éclaire la plupart des interventions ${ }^{9}$ et que cette communauté de valeurs repose avant tout sur l'origine sociale des officiers, il est permis de s'interroger sur la composition et les orientations de cette classe moyenne. Or, si l'on voit bien que la bourgeoisie en constitue la fraction la plus importante, l'article de Nun ne dit pas grand-chose de ses orientations ou intérêts : elle n'a pas de vocation hégémonique et ne remet pas en question les bases matérielles du système oligarchique au sein duquel elle revendique seulement la participation politique et l'égalité des chances. Même lors de la phase de substitution des importations, la classe moyenne, prisonnière de ses intérêts économiques immédiats, n'entre pas en conflit radical avec l'oligarchie, provoquant ainsi une industrialisation sans révolution industrielle, sapant les fondements de l'hégémonie sans cependant la détruire ${ }^{10}$.

Par les militaires, la classe moyenne interviendrait donc tantôt pour élargir sa participation et satisfaire certaines revendications du prolétariat urbain, tantôt pour s'opposer aux gouvernements tentés de s'appuyer un peu trop sur les classes populaires pour apporter les changements structuraux nécessaires au redémarrage de l'économie. Or, tout comme Irigoyen, Perón sera chassé du pouvoir par l'armée très peu de temps après avoir obtenu son succès électoral le plus large, ce qui suppose un soutien au moins partiel de la classe moyenne. On serait tenté dès lors de remettre en question soit la signification des interventions proposée par Nun, soit l'unité de cette classe moyenne aux frontières imprécises et dont, manifestement, certaines fractions peuvent être plus liées à l'urbanisation qu'à l'industrialisation, ou encore être sur le déclin alors que d'autres sont en expansion, etc. $\mathrm{Ne}$ serait-il pas plus avisé de considérer la pluralité des groupes et courants au sein de l'armée et de la classe moyenne ainsi que les liens qui peuvent se tisser entre les uns et les autres, mais aussi entre certains militaires et les autres classes d'une formation sociale? On se demanderait alors, avec Dario Canton, comment, à des moments critiques, certains secteurs de l'armée s'activent, tandis que d'autres demeurent passifs, comme cela se produisit lors du renversement de Perón ${ }^{11}$.

8. Voir en particulier José de Imaz, Los que mandan, Buenos Aires, Editorial Universitaria, 1969 , p. 52 sqq. On sait aussi que les principaux dirigeants militaires brésiliens provienaent de la classe moyenne des petites villes de l'intérieur.

9. J. Nun, "The Middle Class Military Coup », in Claudio Veliz (édit.), op. cit., p. 104. Il faut rappeler que lauteur considère uniquement les pays où la classe moyenne est très développée. Une étude historique sur le Pérou montrerait au contraire que l'armée a constitué un canal de mobilité pour la classe moyenne entre 1890 et 1950 , tout en restant politisée en faveur de l'oligarchie. Voir Liisia North, "Civil-Military Relation in Argentina, Chile and Peru ", Politics of Modernization Studies (Berkeley, University of California), no 2, p. 48-51 et p. 59.

10. J. Nun, "The Middle Class Military Coup », in Claudio Veliz (édit.), op. cit., p. 78 sqq. et p. 86 sqq.

1 l. Military Interventions in Argentina : 1900-1966, Documento de trabajo, Instituto 'Torcuato Di Tella, Centro de investigaciones sociales, 1967, p. 8. 
Car l'unité organisationnelle de l'armée recouvre souvent des luttes entre fractions politisées qui sollicitent l'appui des militaires aux préoccupations plus strictement professionnelles ou corporatistes. De ces divisions, l'armée brésilienne offre un exemple très clair, établi sur une assez longue période : c'est elle qui permet à Vargas d'accéder au pouvoir en 1930 ; plus tard, on trouve des officiers nationalistes disputant à la tendance adverse les élections pour la présidence du Club militaire ; à plusieurs reprises, enfin, le soutien plus ou moins tacite de ces mêmes groupes pèse lourd dans la réalisation des mesures les plus progressistes des gouvernements et dans le maintien de la légalité, jusqu'à l'épuration qui suit le coup de 1964. Dans bien des cas, il semble donc que le sens d'une intervention dépende des rapports de force entre les courants plus ou moins politisés de l'armée, compte tenu des appuis externes - en termes de légitimation - et internes dont chacun bénéficie.

Mais sans doute la faiblesse principale de la thèse de Nun réside-t-elle dans le décalage entre la nature du phénomène observé et l'interprétation proposée : l'auteur considère les coups d'État réussis et compare leurs résultats avec les aspirations de la classe moyenne. Cependant, son interprétation, qui renvoie à l'origine sociale du militaire et à l'armée comme voie privilégiée de mobilité sociale, n'appelle en aucune façon cette limitation aux seuls soulèvements réussis. Elle devrait au contraire pouvoir également s'appliquer aux échecs. Or, certaines données procurent un fondement à l'hypothèse d'une orientation différente pour les coups avortés et pour ceux qui produisent des conséquences politiques observables : sur les trente et une révoltes militaires qui secouent l'Argentine entre 1900 et 1966 , onze sont favorables aux partis populaires ${ }^{12}$, mais échouent toutes, tandis que sur les dix qui s'opposent aux masses, deux seulement avortent, et encore bien sous Perón ${ }^{13}$.

Même si la notion «favorable aux tendances populaires 》 peut viser des alliances de classes différentes dans la période envisagée et si elle n'indique pas exactement la position de la classe moyenne dans le conflit, deux conclusions paraissent s'imposer, pour l'Argentine du moins : en considérant seulement les coups d'État réussis, on favorise systématiquement ceux qui se font contre les masses et l'on ne peut conclure sans risque d'erreur sur les orientations des militaires; d'autre part, la signification objective des interventions ne peut être saisie qu'à partir des rapports de force au niveau de la formation sociale et de leur articulation avec les conflits de tendance au sein de l'armée, dont la formation de loges ou de cellules de partis sont une des expressions les plus achevées. Bref, si les militaires facilitent l'accès puis le maintien de la classe moyenne au pouvoir politique, c'est que ces secteurs moyens jouent déjà un rôle important dans l'activité économique et qu'ils forment la majorité de la population urbaine dans les pays envisagés; l'origine sociale des officiers pèserait finalement peu dans la balance.

Mais ne convient-il pas de faire éclater la notion de classe moyenne et surtout de rejeter l'idée de la bourgeoisie comme fraction de cette classe ?

12. Dario Canton entend par là des coups reliés à l'élargissement de la participation populaire, à l'augmentation de la conscience syndicale et de la capacité organisationnelle des classes laborieuses : "Revolución argentina de 1966 y proyecto nacional », Revista latinoamericana de sociologia, $\mathrm{n}^{\circ} 3,1969, \mathrm{p} .526$.

13. Les dix tentatives restantes ne sont pas classables suivant le critère retenu. 


\section{ALAIN JOXE OU LES OFFICIERS COMME FRACTION DE LA BUREAUCRATIE URBAINE}

Dans un petit livre stimulant et bien documenté consacré aux militaires dans le système politique chilien, Alain Joxe évoque les raisons essentiellement politiques pour lesquelles, bien que détentrice du pouvoir, la bourgeoisie latinoaméricaine continue de se définir comme classe moyenne ${ }^{14}$ : associée à l'oligarchie, vu le contrôle exercé par celle-ci sur les clientèles électorales traditionnelles, mais associée majeure, la bourgeoisie industrielle s'identifie au centre de gravité statistique de l'alliance de classe qu'elle domine, c'est-à-dire aux couches moyennes bureaucratiques urbaines ${ }^{15}$. Pour cet auteur, la nouvelle classe hégémonique représente donc aussi les intérêts de la nouvelle bureaucratie urbaine dont font partie les officiers ${ }^{16}$.

Importante par les précisions qu'elle tente d'apporter sur les rapports entre les militaires et la classe moyenne ainsi que par le cadre de domination impérialiste à l'intérieur duquel elle envisage le rôle politique de l'armée, protectrice des investissements privés, cette recherche aboutit néanmoins à des conclusions fort contestables. Malgré son déroulement quelque peu décousu, la pensée de l'auteur peut être résumée en quelques propositions :

a) Le Chili est, comme les autres pays latino-américains, une formation sociale dépendante ;

b) Le pouvoir d'État, détenu de façon prépondérante par la bourgeoisie industrielle, est institué avec l'aide de l'armée, elle-même dépendante (achats d'armes, aide militaire ${ }^{17}$, entraînement) et garante des investissements étrangers ;

c) La bourgeoisie, dont les intérêts sont liés à la situation de dépendance ${ }^{18}$, développe une classe moyenne comme masse d'appui, ce qui correspond aux intérêts des militaires, partie de la bureaucratie d'État;

d) La décomposition récente de la discipline militaire doit être reliée à la rupture de l'alliance de classe dominée par la bourgeoisie et elle reflète les divergences d'intérêt au sein de la « classe moyenne »;

e) Au sein de celle-ci le comportement spécifique des militaires peut s'inspirer de leur situation la plus immédiate de salariés non productifs, tandis que les intérêts divergent, au sein de l'armée, sur la question des strates à favoriser à l'intérieur de la classe moyenne (hauts ou bas revenus) et sur la politique d'achats d'armements. Cependant, de façon moins immédiate, l'armée a intérêt à éviter la guerre civile, ce qui peut l'amener à encourager une politique de développement, et ce malgré sa nature de catégorie sociale non productive. L'institution militaire peut même, à partir de son pouvoir spécifique de négociation, cesser de se comporter

14. Alain Joxe, las Fuerzas armadas en el sistema politico de Chile, p. 126 sqq.

15. Ibid., p. 131 sqq.

16. Ibid., p. 133.

17. S'appuyant sur des documents américains, l'auteur montre comment l'aide militaire au Chili varie d'un minimum d'environ $4 \%$ des dépenses annuelles de la Défense nationale à environ $30 \%$ de ce même budget en période critique (1963, période préélectorale où le F.R.A.P. apparaît comme un possible vainqueur). Cf. ibid., p. 106 sqq.

18. Cette proposition n'est pas développée. Pour l'auteur, les investissements étrangers provoquent l'expansion et renforcent le pouvoir économique de l'Etat en même temps que sa dépendance (cf. ibid., p. 115). Ce point doit être mis en relation avec l'alliance de classe dans laquelle la bourgeoisie contrôle la distribution des richesses, mais non le crédit ou l'inflation, toujours aux mains de l'oligarchie financière (cf. ibid., p. 113). 
comme une partie de la classe moyenne salariée et entreprendre une politique de développement par coercition dont le poids repose sur les salariés et les tranches basses de revenus.

Cette dernière partie de la thèse d'Alain Joxe paraît tout à fait critiquable ; elle illustre parfaitement les limites d'une analyse où le comportement politique de l'armée est rapporté directement à la position de classe de ses membres. En effet, des quatre critères retenus par l'auteur pour décomposer la classe moyenne en fractions et situer les militaires parmi elles ${ }^{19}$, deux seulement s'appliquent en toute rigueur : les militaires sont salariés et non productifs. On a vu cependant que ni l'une ni l'autre de ces caractéristiques n'est suffisante pour rendre compte des multiples interventions possibles. Les critères retenus peuvent tout au plus servir à effectuer une nomenclature de différents types de politiques de développement.

Par-delà cette critique immédiate, c'est l'analyse de l'armée comme fraction de classe sociale, d'appartenance ou d'origine, qui doit être contestée : il ne paraît pas possible de déduire directement, avec un minimum de précision, les orientations des militaires des intérêts plus ou moins problématiques dérivés de leur position de classe. Il faut, au contraire, procéder à une analyse en termes d'appareil d'État et tenter d'articuler les stratégies et les rapports de force entre les fractions politisées de l'armée et le déroulement de la lutte des classes dans la société.

L'auteur avait engagé sa recherche dans une voie féconde : il avait souligné la dépendance extérieure de l'armée ; à partir des variations du montant de l'aide américaine, il avait mis en évidencè la fonction de l'appareil militaire comme gardien des investissements étrangers et comme agent de constitution de l'État dominé; il avait établi un lien entre la professionnalisation des forces armées et les ventes d'armements effectuées par les métropoles selon les exigences de leur propre développement. Cependant, en mettant trop exclusivement l'accent sur les facteurs externes, sur l'impérialisme plus que sur le dynamisme spécifique, à l'intérieur de certaines limites, des formations sociales dépendantes, l'auteur courait le risque de prêter un caractère quasi instrumental à l'armée par rapport au projet de domination international et de se priver des moyens d'interprétation de l'ensemble des phénomènes observables. Aussi cette approche fut-elle délaissée en cours de route, au profit de l'hypothèse de l'appartenance des militaires à la bureaucratie d'État, fraction de la classe moyenne.

Peut-être cette impasse aurait-elle été évitée si l'auteur avait envisagé l'exposition de l'armée à un double pouvoir de classe : celui de la formation dominante et celui, relativement décalé, qui prévaut dans les formations dépendantes. Telle est la thèse défendue dans cet article, qui nous conduira à envisager tout d'abord l'armée comme appareil d'État, pour analyser ensuite l'incidence des mécanismes de l'aide étrangère sur le rôle politique de cet appareil, dans le cadre d'une situation générale de dépendance.

19. Salariés - non salariés ; secteurs productifs - non productifs; revenus moyens supérieurs - moyens inférieurs; lien avec les groupes dominants nationaux - dominants étrangers (cf. Alain Joxe, las Fuerzas armadas en el sistema politico de Chile, p. 139-140). 


\section{L'ARMEEE COMME APPAREIL D'ÉTAT}

Le concept d'appareil d'Etat appliqué à l'armée renvoie à son appartenance à l'État et au pouvoir de classe qui s'y exerce ${ }^{20}$. Car, comme l'a bien souligné Poulantzas à propos de ces appareils en général, considérée en elle-même, l'armée n'a guère d'intérêts spécifiques et pas davantage de pouvoir propre : au même titre que l'État dont elle est un des rouages, elle concentre du pouvoir émanant des véritables forces sociales : les classes et leurs fractions.

C'est pourquoi, dans le cadre d'une hégémonie bien assise, le problème du contrôle de l'armée se résout facilement. D'une part, l'appareil militaire est une pièce trop centrale de l'État pour que la classe hégémonique ne vise pas à affirmer en lui et à travers lui son pouvoir. D'autre part, la dominance de classe est suffisamment marquée pour que l'inégalité des pouvoirs s'exprime de façon non équivoque dans le comportement militaire, quelle que soit l'origine sociale des officiers supérieurs.

Cependant, cette conformité de l'armée aux intérêts de la classe dominante se réalise généralement par la médiation de normes internes régissant le recrutement, la formation et la promotion des militaires, et de règles externes définissant, dans le cadre de certaines limites, le pouvoir d'intervention de l'armée sur les autres appareils ainsi que sur les citoyens. L'établissement d'un tel système juridique est en effet l'un des traits essentiels de la situation d'hégémonie, laquelle ne peut reposer exclusivement sur l'imposition directe et brutale des intérêts de classe. Grâce à ce système normatif, les décisions des militaires sont rationnelles dans le cadre de la spécialisation fonctionnelle qui leur est assignée et s'inscrivent du même coup dans la ligne des intérêts hégémoniques.

Bref, le système normatif institué désigne les détenteurs des ressources de pouvoir disponibles ainsi que la destination de celles-ci et les limites de leur utilisation. Dans cette perspective, la montée d'une classe sociale ou la situation d'équilibre des forces en présence doivent être envisagées comme le développement d'un second pouvoir, de telle sorte qu'il y a rupture du quasi-monopole ancien et que peuvent se créer des réseaux parallèles de distribution des instruments matériels, coercitifs ou symboliques de pouvoir.

Dans de telles situations d'équilibre des forces, l'armée peut jouer un rôle important dans l'établissement des conditions d'accession d'une classe montante à l'hégémonie. L'origine sociale des cadres de l'organisation militaire peut évidemment constituer un facteur de cette intervention mais, de façon plus générale, c'est l'orientation idéologique des dirigeants ou des fractions militaires qui est déterminante. Nous posons en outre l'hypothèse que dans ce cas, l'idéologie prend généralement appui sur une sorte de relais organisationnel, effectuant la jonction entre les représentants de la classe montante et certains militaires plus ou moins

20. Pour une théorie des appareils d'Etat, voir Nicos Poulantzas, Pouvoir politique et classes sociales, Paris, Maspero, 1968, notamment les chapitres finals sur la bureaucratie. Du même auteur, on peut consulter également Fascisme et dictature, Paris, Maspero, 1970, en particulier la septième partie sur l'Etat fasciste. On sait que, de façon tout à fait générale, la notion d'appareil d'Etat renvoie à ces « institutions» dans lesquelles s'incarnent le pouvoir et la dornination de classe et qui, du fait de leur appartenance à l'Etat, ont pour fonction d'assurer la cohésion de la formation sociale, soit par la répression, soit par l'idéologie. Voir Althusser, la Pensée, juin 1970, et Bourdieu et al., la Reproduction. 
sensibilisés à leurs aspirations. Le contrôle par ces officiers d'une zone d'incertitude, en termes de pouvoir, peut leur assurer un avancement accéléré au sein de l'armée et, simultanément, les amener à établir les conditions de l'accession à l'hégémonie de cette classe à laquelle ils sont redevables de leur carrière militaire et politique.

Le passage de Perón au ministère du Travail est trop célèbre pour devoir être évoqué à l'appui de cette hypothèse. Par contre, la prise du gouvernement bolivien par German Busch en 1937, et l'orientation populiste de sa politique sont moins connues; or, ce jeune général, issu de la classe moyenne ${ }^{21}$ et qui doit sa promotion rapide à sa conduite héroïque au front, prend le pouvoir après avoir été porté à la tête de la Légion des anciens combattants, c'est-à-dire de ces anciennes recrues de la guerre du Chaco, revenus tant bien que mal à la vie civile et qui constituent la fraction organisée d'une masse mobilisée beaucoup plus importante.

Cependant, le type d'explication que l'on vient d'esquisser vise une situation particulière d'équilibre des forces dans une période de transition et ne pourrait donc s'appliquer à l'ensemble des coups militaires à signification politique. Le cas de Perón évoqué précédemment est un exemple parmi d'autres de l'influence considérable des facteurs externes : les liens du futur président argentin avec l'ambassade d'Allemagne sont notoires, de même que ceux du général Villarroël qui s'impose en Bolivie à peu près à la même époque. Il faut par conséquent analyser l'armée comme appareil d'un État dépendant, pour ensuite chercher à préciser les relations particulières qu'elle entretient avec cet État.

En d'autres termes, nous voudrions développer l'hypothèse selon laquelle l'explication du comportement politique des militaires doit tenir compte de deux aspects essentiels : les États latino-américains sont dans une position de dépendance et, ce qui n'est pas tout à fait la même proposition, leurs armées sont un objet très privilégié de l'aide étrangère; elles sont donc en relation plus ou moins directe avec un État étranger, par l'entremise de l'appareil militaire de celui-ci. La notion de dépendance renvoie aux contradictions spécifiques opérant dans les formations de la périphérie et aux luttes sociales qui s'y déroulent; l'accent mis sur l'aide étrangère conduit au rejet de la conception de l'armée latino-américaine comme appareil incarnant un seul pouvoir d'État, national et spécifique.

\section{DÉPENDANCE ET CONTRADICTIONS}

Le concept et la problématique de la dépendance permettent de considérer les phénomènes politiques des formations sociales dans leur spécificité et dans leur

21. German Busch était le fils d'un immigrant allemand et d'une Bolivienne. Sur les tentatives populistes qui précédèrent la révolution nationale de 1952, en Bolivie, et qui furent toutes dirigées par des militaires, on peut consulter notamment les recherches historiques de Herbert S. Klein, Origenes de la revolucion nacional boliviana, La Paz, Editorial Juventud, 1968, ainsi que, du même auteur, "German Busch and the Era of "Military Socialism " in Bolivia ", in The Hispanic American Historical Review, vol. XLVII, no 2, 1967, p. 166-184. Voir également "David Toro and the Establishment of "Military Socialism " in Bolivia ", publie par cet auteur in The Hispanic American Historical Review, vol. XLV, $n^{\circ}$ 1, février 1965 , p. 25-52. 
conditionnement par l'appartenance au marché international. Autrement dit, il n'est pas pertinent d'analyser la dynamique des formations sociales latino-américaines à partir des seuls facteurs internes et sans tenir compte de la signification des modifications du système capitaliste international dont ils font partie ; inversement, une étude qui tirerait argument de la dépendance pour n'envisager que des facteurs externes d'explication et leurs conséquences immédiates ne pourrait rendre compte de la spécificité du rôle de l'État et de la lutte des classes dans ces formations ${ }^{22}$. Le concept de dépendance vise donc l'articulation de ces deux niveaux de relations, externes et internes.

Les freins au développement du mode de production capitaliste dans les formations dépendantes, le développement du sous-développement comme on a pu dire, se traduisent par la constitution de blocs au pouvoir et de rapports de force particuliers, par des intérêts politiques et des idéologies spécifiques des classes dominantes, etc. ${ }^{23}$. Bref, les États du centre et de la périphérie peuvent être plus ou moins décalés les uns par rapport aux autres, dans la mesure où ils organisent la domination de classe dans des formations distinctes.

Cardoso pose donc bien le problème, lorsqu'il affirme que « la contradiction entre les classes dans les situations de dépendance inclut des contradictions spécifiques entre la nation [l'État] et l'impérialisme et entre les intérêts locaux des classes dominantes et leur caractère internationalisant ${ }^{24}$ 》.

Bref, si l'on veut éviter de réduire un système politique et social à un ordre économique, il faut procéder à l'analyse des forces spécifiques engendrées dans les formations dépendantes par la domination économique internationale et ce, en tenant compte des modalités historiques de la liaison établie avec le marché capitaliste mondial ${ }^{25}$. En outre, la relation de dépendance envers les formations du centre fait que la contradiction de classe qui se pose au niveau du système capitaliste tout entier passe par des contradictions spécifiques sur le plan national et par celles qui résultent de l'existence d'États nationaux ${ }^{26}$.

22. Voir Fernando H. Cardoso, Teoria da dependência ou Análises concretas de situaçôes de dependência, texte préliminaire présenté en vue de discussion au séminaire de la FLASCO, novembre 1970 , en réponse à un texte de F. C. Weffort, Notas sobre a «teoria da dependência» : teoria de classe ou idéologia nacional?

23. Par exemple, l'existence d'une masse paysanne considérable et le contrôle politique exercé sur elle par les grands propriétaires terriens peuvent conduire à des alliances de classe spécifiques (bourgeoisie industrielle - oligarchie) à certains stades du développement dépendant. Cardoso a examiné cette question dans a Les obstacles structurels et institutionnels au développement ", Sociologie et Sociétés, vol. 2, n 2, novembre 1970.

24. Fernando H. Cardoso, Teoria da dependência..., p. 7. Cette thèse est controversée. Pour Samir Amin par exemple, la contradiction est entre la bourgeoisie mondiale et le prolétariat mondial parce que le capitalisme est devenu un système mondial. La bourgeoisie de la périphérie n'est pas exploitée mais limitée dans son développement et elle ne peut reporter le pillage dont elle est victime que sur son prolétariat. Voir l'Accumulation d l'échelle mondiale, Paris, Anthropos, 1970, en particulier p. 33 sqq. ; voir aussi F. C. Weffort, Notas sobre a «teoria da dependência \$...

25. Voir F. H. Cardoso, Politique et développement dans les sociétés dépendantes, Paris, Anthropos, 1971, chap II. L'auteur distingue les structures dépendantes dans lesquelles les groupes locaux ont longtemps pu conserver un contrôle sur l'organisation du secteur agricole d'exportation et les économies d'enclave minière où ce contrôle est tombé aux mains des investisseurs étrangers. L'alliance de classe oligarchique et les fonctions de l'Etat sont différentes dans les deux cas, ainsi que la circulation du capital et les possibilités de développement du marché intérieur.

26. F. H. Cardoso, Teoria da dependência..., p. 7-8. 


\section{L'INTERNATIONALISATION DES ARMÉES LATINO-AMÉRICAINES}

La notion d'internationalisation avancée ici est destinée à souligner les liens de toute nature qui unissent les officiers latino-américains aux États-Unis et à leur armée. Grâce à leur position stratégique au sein de l'État et à leur quasi-monopole sur les ressources coercitives, les armées latino-américaines obtiennent une aide étrangère souvent considérable en armes et équipements, en formation idéologique ${ }^{27}$, en techniques spéciales comme la lutte antiguérilla ou l'action civique ${ }^{28}$.

Cette situation n'est pas nouvelle, bien que les formes et le contenu même de l'aide aient passablement changé. Des missions françaises ou allemandes ont joué un rôle primordial dans la professionnalisation des forces latino-américaines dès le début de ce siècle, relayées ensuite par les États-Unis. Ainsi, en Bolivie, une mission française réorganise les programmes d'enseignement dans les écoles militaires entre 1905 et 1909. Dès 1911, une mission allemande lui succède dont le responsable est nommé chef d'état-major de l'armée bolivienne; la dépendance prend alors des formes presque caricaturales avec l'adoption des règlements et uniformes allemands à peine retouchés. La guerre 1916-1918 interrompt l'œuvre entreprise qui reprend en 1921 ; en 1925, le général allemand Kundt dirige le ministère bolivien de la Guerre; lors du conflit avec le Paraguay, en 1932, ce même militaire est sollicité par le Congrès bolivien pour devenir commandat en chef des forces armées, mission pour laquelle il reçoit pratiquement carte blanche ${ }^{29}$. Avec le déplacement de la prépondérance de l'Europe vers les États-Unis, c'est vers ce dernier pays que se tourneront les dirigeants de la Révolution nationale : dès 1956, des Américains font office de conseillers militaires en matière d'entraînement, de doctrine militaire, de standardisation de l'équipement et de son emploi effectif ${ }^{30}$. Enfin, pour clore une énumération qui risquerait de devenir fastidieuse, les conseillers américains « collaborent » et enseignent dans plusieurs des écoles destinées aux militaires boliviens, tandis que, selon une source américaine, le montant de l'aide en 1965 s'élèverait à $16,9 \%$ du budget de la Défense ${ }^{31}$.

L'intervention d'un État étranger n'est sans doute aussi massive dans aucun autre secteur d'activité ${ }^{32}$. Bien entendu, dans des pays plus industrialisés et au nationalisme plus ombrageux, les États-Unis sont amenés à une assistance technique moins voyante et qui s'adresse alors moins à l'organisation dans son

27. Théorie des frontières idéologiques, extension de la notion d'ordre public aux facteurs sociaux, politiques, etc., qui le conditionnent.

28. Les réalisations de Barrientos dans l'Action civique furent à l'origine de sa popularité auprès des masses paysannes boliviennes.

29. Voir Alfonso Camacho Peña, "Los militares en la política boliviana », Aportes, $n^{\circ} 22$, octobre 1971, p. 41-95. Egalement Herbert S. Klein, Origenes de la revolución nacional boliviana, passim, et notamment le chapitre vi sur la guerre du Chaco.

30. Voir Alfonso Camacho Peña, * Los militares en la politica boliviana s, Aportes, no 22, octobre 1971, p. 74-75. L'auteur'se réfère à l'U.S. Army Handbook for Bolivia, 1963, p. 695 .

31. Ce pourcentage est cité par Joxe (los Fuerzas armadas, en el sistema politico de Chile, p. 104), qui le reprend du Survey of the Alliance for Progress et qui critique la base statistique qui a servi à l'établir. Des données plus générales ne sont pas disponibles. actuellement.

32. L'enseignement supérieur est un autre terrain de prédilection de l'aide américaine, avec des conséquences importantes sur le plan interne et du point de vue de ses fonctions dans la société. Voir Luis Scherz García, "Algunos aspectos disfuncionales de la ayuda internacional y el papel de la Universidad en el cambio social de America latina", America Iatina, $n^{\circ} 3$, juin-septembre 1967 , spécialement p. 67 sqq. 
ensemble qu'à des individus : stages ou études à Panama ou aux États-Unis, par exemple. Le cas du Chili évoqué au début de cet article montre cependant que, même pour des pays moins démunis, l'aide matérielle reste très considérable, surtout dans les périodes d'incertitude politique.

Cette internationalisation des armées latino-américaines nous paraît importante pour comprendre leur autonomie, toute particulière pour un appareil aussi central de l'État, à l'égard du pouvoir d'État local. Ce dernier a en effet pour fonction de réaliser les intérêts politiques de la classe dominante, compte tenu de ses rapports de force avec les autres classes et de la nécessité de maintenir la cohésion sociale. Le rôle stratégique à cet égard de l'appareil militaire rend impérative l'existence d'une armée bien équipée et satisfaite de son statut socio-économique, donc financée de l'extérieur. Mais cette même relation internationale constitue un facteur essentiel de l'autonomisation de cet appareil et le place en quelque sorte dans une position d'arbitrage entre les États périphérique et central.

Les classes en présence et leurs rapports de force étant différents dans les formations capitalistes du centre et de la périphérie, ainsi que la combinaison des modes de production et de leurs stades, les intérêts politiques de l'une et l'autre classe dominante peuvent présenter certaines contradictions ou être nettement décalés. C'est ce qu'exprime, pour nous, la notion de dépendance selon laquelle, si les changements dans les sociétés dominées se réalisent en réponse à des incitations et transformations externes, ils n'en sont pas pour autant les effets purs et simples, non médiatisés, en raison des particularités des classes en présence et de leur lutte.

Bref, au sein de l'armée, le pouvoir d'État local trouve un contrepoids éventuel dans l'influence exercée par un pouvoir d'État étranger dans le cadre général de la dépendance et de l'assistance militaire, celle-ci comme élément fonctionnel pour le maintien de celle-là. Et, dans les deux cas, il s'agit bien d'un pouvoir de classe, correspondant aux intérêts politiques des classes dominantes des deux formations sociales.

\section{CONCLUSION PROVISOIRE}

Dans cet article, plusieurs types d'analyse ont été soumis à la critique. L'interprétation du comportement militaire par l'identification pure et simple à une classe, et sans considération pour la position dépendante des sociétés latino-américaines, ne peut en effet être retenue. Pas davantage, le modèle d'explication fondé sur l'impérialisme n'est capable de rendre compte avec précision des comportements contradictoires de l'armée et on ne s'y soucie pas assez des médiations internes.

Très vite, il est apparu que l'armée devait être traitée comme un appareil d'État, mais dont le mécanisme de contrôle et d'orientation serait relativement faussé : sa relation avec un pouvoir étranger produit ses effets à l'intérieur même de l'appareil au plan des orientations idéologiques légitimes et des rapports de force. 
Cette double « allégeance» de l'armée ne repose pas sur des rapports identiques avec les deux pouvoirs d'État, c'est évident. Elle permet cependant de comprendre l'autonomie remarquable des forces militaires à l'égard du pouvoir d'État local. En outre, la thèse de la double allégeance offre un principe d'analyse utile, à partir duquel il est possible de reconstruire les orientations communes au sein de l'armée et les points sur lesquels les fractions peuvent s'affronter dans des cadres donnés de dépendance structurelle, à des phases ou des stades déterminés de développement.

Dans cette ligne de raisonnement, on peut poser l'hypothèse de l'unité idéologique relative de l'appareil chaque fois que les contradictions entre l'État national et l'État impérialiste sont relativement faibles, et inversement.

Le problème des interventions politiques n'est cependant pas épuisé par une approche en termes d'orientations idéologiques. À cet égard, la rupture, par le mécanisme de l'aide étrangère, du monopole de l'État national en matière de distribution et d'orientation des instruments de pouvoir, constitue un aspect primordial de l'autonomie dont jouissent actuellement les militaires latino-américains et de leur capacité d'intervention, même si celle-ci repose le plus souvent sur le consentement plus ou moins actif de certaines classes ou fractions.

On ne pourrait, en effet, sous-estimer les conséquences de la création au sein des forces armées, où le niveau de formation socio-politique demeure parfois très bas, d'un réseau d'hommes pourvus d'une idéologie relativement structurée et disposant à l'occasion de ressources matérielles non négligeables. Ainsi, en Bolivie, ces officiers « internationalisants » ont joué un rôle essentiel dans les trois soulèvements militaires contre le régime national du général Torres. Dans une formation sociale où la révolution nationale de 1952 a libéré des forces considérables, sans réellement affecter le lien de dépendance structurelle, les contradictions entre les États national et impérialiste tendent à être relativement aiguës; d'où les orientations contradictoires de l'armée, ses divisions. Cependant la situation des forces en présence n'est pas identique : la fraction internationalisante jouit d'un avantage assez net à l'intérieur de l'appareil et peut généralement compter sur les syndicats de paysans contrôlés par l'État; par contre, les nationalistes ne peuvent se hisser et se maintenir au gouvernement qu'avec l'appui, qui est loin d'être inconditionnel, des organisations de travailleurs et d'étudiants. La création de relais organisationnels plus ou moins stables entre les fractions militaires et les classes susceptibles d'orienter et d'appuyer leur action constitue donc l'une des conditions de la consolidation d'un régime national et populaire.

Le coup d'Êtat des militaires brésiliens peut également s'analyser suivant les principes proposés dans cet article. Le gouvernement actuel établit les conditions d'émergence d'un nouveau bloc au pouvoir fondé sur l'industrialisation restrictive et la fin des politiques populistes de redistribution des richesses. Les généraux, à vocation internationale marquée et partisans des frontières idéologiques, favorisent, au plan intérieur, la montée d'une fraction de classe liée au développement de l'industrie lourde et des grandes sociétés multinationales installées au pays et produisant pour le marché intérieur.

L'accession des militaires au pouvoir clôt donc, au Brésil, la période de substitution des importations et met fin, par une répression parfois barbare, à une 
politiçue populiste de redistribution des ressources et d'élargissement du marché, qui ne correspond plus aux intérêts des secteurs les plus avancés de l'économie ${ }^{33}$. L'action gouvernementale profite donc à la bourgeoisie industrielle la plus moderne et à vocation internationale; celle-ci voudrait transformer l'Etat en instrument efficace d'une politique économique axée sur la concentration des capitaux. Les propriétaires terriens, qui appuyaient le coup d'État par antipopulisme, ne sont finalernent ni les bénéficiaires ni les promoteurs des transformations en cours : leur pouvoir, qui se manifestait surtout sur les plans local et régional, est fortement ébranlé et la politique du gouvernement tend à les enfermer dans le dilemme de la modernisation ou de la disparition comme force politique ${ }^{34}$. L'action entreprise par les dirigeants militaires est donc moderne en ce qu'elle affaiblit le pouvoir de la vieille oligarchie et lève de nombreux obstacles institutionnels à la croissance. Les liens qui ont pu s'instaurer entre certains militaires et des représentants de la bourgeoisie à travers des organes de coordination comme le Groupe permanent de mobilisation industrielle ${ }^{\mathbf{3 5}}$ trahissent d'ailleurs le contenu de classe de la politique brésilienne actuelle. Branchée sur le développement d'une fraction de la bourgeoisie industrielle et sur ce que Cardoso a appelé l'internationalisation du marché intérieur ${ }^{36}$, l'armée favorise sur le plan national la montée de la classe détenant l'hégérnonie dans les formations du centre et tend à réduire le décalage entre État national et État impérialiste par la mise en place d'un nouveau pouvoir d'État au Brésil.

\section{RÉSUMÉ}

Après avoir montré l'impossibilité d'analyser le rôle politique des militaires latino-américains à partir de leur origine sociale ou de leur appartenance à la bureaucratie urbaine, l'auteur propose de considérer l'armée comme appareil d'État. Cependant, dans une situation de dépendance, l'aide militaire étrangère vient perturber les mécanismes de contrôle de la classe dominante, sur l'appareil militaire. L'examen de ces deux aspects - appareil d'État et aide étrangère - permet de comprendre la marge d'autonomie de l'armée ainsi que le sens et les limites ale son rôle politique, compte tenu des classes et de leurs rapports dans les formations latino-américaines.

\section{ABSTRACT}

[The Military Apparatus in Latin American Dependent Social Organization] After having shown the impossibility of analyzing the political role of the Latin American military force

33i. Une première dégradation de la politique nationale, sur le plan économique et populaire, sur le plan sociopolitique, s'était déjà produite après la mort de Vargas, avec l'avènement de: Kubitschek. Voir O. Ianni, $O$ colapso do populismo no Brasil. Pour les problèmes soulevés par la politique de substitution des importations au Brésil, on peut consulter Celso Furtado, les Etats-Unis et le sous-développement de l'Amérique latine, Paris, Calmann-Lévy, 1970 , p. 137 sqq.

34. Sur l'alliance de classe qui a porté les militaires au pouvoir et les rapports entre la bourgeoisie et les propriétaires terriens, voir Roberto Decio de las Casas, « L'Etat autoritaire ; essai sur les formes actuelles de la domination impérialiste ", l'Homme et la société, no 18 , octobre-décembre 1970 , p. 99-111.

35. Créé en avril 1964, ce groupe tend à harmoniser la production de matériel et d'équipement militaire ainsi que la recherche industrielle avec les besoins de l'armée. Voir Octavio Ianni, $O$ colapso do populismo no Brasil, p. 205 sqq. L'auteur considère cette création comme l'institutionnalisation du complexe industriel militaire au Brésil.

36. C'est-à-dire que dans les pays les plus industrialisés de la périphérie, le secteur moderne tend à se soumettre les autres secteurs et est régi par des lois de capitalisation, de productivité et de marché semblables à celles qui prédominent au centre. Voir F. H. Cardoso, Politique et développement dans les sociétés dépendantes, p. 156 sqq. 
according to its social origins or its belonging to the urban bureaucracy, the author proposes to consider the army as an apparatus of the state. However in a situation of dependance, foreign military aid disturbs the control mechanisms of the dominant class, on the military apparatus. An examination of its two aspects - apparatus of the state and foreign aid allows one to understand the margin of the army's autonomy as well as the significance and the limits of its political role, having regards to the classes and their relationships in Latin American social organization.

\section{RESUMEN}

[Los aparatos militares en las formaciones sociales dependientes de América latina] Después de haber mostrado la imposibilidad de analizar el papel político de los militares latinoamericanos a partir de su origen social o de su pertenecia a la burocracia urbana, el autor propone que se considere la armada como aparato de Estado. Por otro lado, en una situación de dependencia, la ayuda militar extranjera viene a perturbar los mecanismos de control de la clase dominante, respecto al aparato militar. El examen de estos dos aspectos - aparato de Estado y ayuda extranjera - permite comprender el margen de autonomía de la armada así como el sentido y los límites de su papel político, teniendo en cuenta las clases sociales y sus relaciones en las formaciones latinoamericanas. 